\title{
Variations écologiques et chorologiques de la végétation macrophytique des rivières acides du Massif armoricain et des Vosges du Nord (France) \\ Ecological and chorotogical variations of macrophytic vegetation in acid rivers of Armorican Massif and Northern Vosges (France)
}

\author{
J. Haury et S. Muller
}

Volume 4, numéro 4, 1991

URI : https://id.erudit.org/iderudit/705111ar

DOI : https://doi.org/10.7202/705111ar

\section{Aller au sommaire du numéro}

\section{Éditeur(s)}

Université du Québec - INRS-Eau, Terre et Environnement (INRS-ETE)

\section{ISSN}

0992-7158 (imprimé)

1718-8598 (numérique)

\section{Découvrir la revue}

Citer cet article

Haury, J. \& Muller, S. (1991). Variations écologiques et chorologiques de la végétation macrophytique des rivières acides du Massif armoricain et des Vosges du Nord (France). Revue des sciences de l'eau / Journal of Water Science, 4(4), 463-482. https://doi.org/10.7202/705111ar

\section{Résumé de l'article}

La végétation macrophytique des rivières acides d'Europe occidentale présente une grande unité, marquée par la constance d'un ensemble floristique de base, dont certaines espèces (Callitriche hamulata, Myriophyllum alterniflorum, Potamogeton polygonifolius, $P$. alpinus, etc) sont propres à ces eaux acides, d'autres (Callitriche platycarpa, Sparganium emersum, Elodea canadensis, Potamogeton crispus, etc) étant communes avec les eaux calcaires. Cet ensemble subit des variations écologiques et chorologiques :

L'augmentation du niveau trophique des eaux conduit d'une végétation oligotrophe (différenciée par P. polygonifolius, Juncus bulbosus, etc) à un groupement mésoeutrophe (caractérisé par Callitriche obtusangula, Nasturtium officinale, etc). Ainsi, quatre types de phytocénoses ont pu étre distingués dans les Vosges du Nord en fonction de ce paramètre. D'autre part, une pollution organique localisée, par exemple par une pisciculture en Bretagne, provoque une augmentation des espèces eutrophes ( $C$. obtusangula) et une régression des taxons plus oligotrophes (Scapania undulata).

L'alternance de biotopes courants et lents se traduit par un remplacement de phytocénoses : ainsi, en Bretagne, Oenanthe crocata et Ranunculus penicillafus sont associées sur les radiers, alors que Nuphar lutea et S. emersum caractérisent les mouilles. De plus, une diminution de l'éclairement permet la présence de groupements bryophytiques spécifiques.Les principales variations chorologiques entre les deux territoires correspondent à un enrichissement des zones atlantiques en macrophytes euatlantiques ou méditerranéoatiantiques, comme $O$. crocata ou Apium inundatum, qui manquent dans les régions médioeuropéennes. En outre, selon les territoires biogéographiques, l'écologie de certaines espèces peut varier (par exempte $S$. emersum, R. peltatus, $P$. polygonifolius).

Les macrophytes permettent donc, à l'instar des invertébrés benthiques, l'élaboration de biodiagnostics des eaux courantes. 


\title{
Variations écologiques et chorologiques de la végétation macrophytique des rivières acides du Massif armoricain et des Vosges du Nord (France)*
}

\author{
Ecological and chorotogical variations \\ of macrophytic vegetation in acid rivers \\ of Armorican Massif and Northern Vosges (France)
}

J. HAURY1 , S. MULLER 2

Reçu le 24 août 1990, accepté pour publication le 17 mai 1991*.

RÉSUMÉ

La végétation macrophytique des rivières acldes d'Europe occidentale présente une grande unité, marquée par la constance d'un ensemble floristique de base, dont certaines especes (Callitriche hamulata, MyriophylIum altermillorum, Potamogeton polygonifolius, P. alpinus, etc) sont propres a ces eaux acides, d'autres (Callitriche platycarpa, Sparganium emersum, Elodea canadensis, Potamogeton crispus, etc) étant communes avec les eaux calcaires.

Cet ensemble subit des variations écologiques et chorologiques :

L'augmentation du niveau trophique des eaux conduit d'une végétation oligotrophe (différenciée par $P$. polygonifolius, Juncus bulbosus, etc) à un groupement mésoeutrophe (caractérisé par Callitriche obfusangula, NasturIium officinale, etc). Ainsi, quatre types de phylocénoses ont pu étre distingués dans les Vosjes du Nord en fonction de ce paramètre. D'autre part, une pollution organique localisée, par exemple par une pisciculture en Bretagne, provoque une augmentation des espèces eutrophes (C. obfusangula) et une régression des laxons plus oligotrophes (Scapania undulata).

L'alternance de biotopes courants et lents se traduil par un remplacement de phytocénoses : ainsi, en Bretagne, Oenanthe crocata el Ranunculus penicilla fus sont associées sur les radiers, alors que Nuphar futea et $\boldsymbol{S}$. emersum caractérisent les mouilles. De plus, une diminution de l'éclairement permet la présence de groupements bryophytiques spécifiques.

1. ENSA Botanique et INRA Ecologie tydrobiologique Rennes, 65 , Route de Saint-Brieuc, 35042 Rennes Cedex.

2. Université de Metz, Laboratoire d'Ecologie, 1, nue des Récollets, 57000 Metz.

Communication présentée au 348 Congrès de l'Association Française de Limnologie, Metz-Nancy, 29-31 mai 1990.

* Les commentaires sont acceptés jusqu'au 15 septembre 1992. 
Les principales variations chorologiques entre les deux territoires correspondent à un enrichissement des zones attantiques en macrophytes euatlantiques ou méditerranéoatlantiques, comme O. crocata ou Apium inundatum, qui manquent dans les régions médioeuropéennes. En outre, selon les territoires biogéographiques, l'écologie de certalnes espèces peut varier (par exemple S. emersum, R. pelfatus, P. polygonifolius).

Les macrophytes permettent donc, à l'instar des invertébrés benthiques, l'élaboration de biodiagnostics des eaux courantes.

Mots clés : rivière acide, macrophyte, qualité de l'eau, indication biologique, chorologie.

For the study of the vegetation of acid rivers in France, two areas have been investigated by the authors : Armorican Massif and Northern Vosges (fig. 1).

This paper has two aims :

- assessing the general trends of macrophytic vegetation : floristic composition and plant communilles, with special reference to the influence of physical and water quality parameters,

- pointing out and interpreting the variations observed between the two regions (chorological differences), and in the watercourses (ecological variations).

Floristlc data and phytosociological surveys (from bryophytes to anglosperm macrophytes) were collected along $50 \mathrm{~m}$. stretches (at least) by the stratified sampling method in the case of large watercourses, and by systematic surveys for smaller ones. Consecutive $50 \mathrm{~m}$. long stretches were used to measure the effect of localized pollution. (The inventory is as precise as possible). A distinction was made between the aquatic communities and riverbankside ones. Al the same time, physical parameters were measured or observed (depth, light conditions, width,...), certain water-quality parameters were measured ( $\mathrm{pH}$, conductivity,...) and water samples were collected for further analysis.

The data collecled are gathered and compared in a table (table 1) showing differences between aquatic and subaquatic species, and between sunlit and shaded stretches. Trophic tendencies of most species are given according to either our observations or the literature.

The main ecological feature is the streaking longitudinal variallon of aquatic species, which agrees with the zones described by ILLIES and BOTOSANEANU: crenon, mitron and potamon. In sunilt areas the succession is dominated by the following species:

Potamogeton polygonifolius, Juncus bulbosus, Glyceria fluitans,...

$\rightarrow$ Ranunculus spp., Callitriche hamulata, Myriophyllum altemillorum,...

$\rightarrow$ Polamogeton crispus, Elodea canadensis,...

Areas in the shade are characterized by bryophyta; the succession is: Scapanla undulata

$\rightarrow$ Ahynchostegium nparioides, fontinalls antipyretica

As for the aquatic species, a longltudinal succession of bankside species exists :

Sparganium erectum, Carex paniculata, Carex rostrata

$\rightarrow$ Phalaris arundinaces

$\rightarrow$ Phalaris arundinacea, Typha latifolia 
The macrophytic vegetation in acid rivers of Western Europe shows a great homogeneity, marked by the constancy of a floristic list, in which some species (Callitriche hamulata, Myriophyllum alterniflorum, Pofamogeton polygonifollus, Potamogeton alpinus, Scapania undulata, etc.) only grow into acid waters, while others species (Callitriche plafycarpa, Sparganium emersum, Elodea canadensis, Potamogefon crispus, efc.) grow also in calcareous waters.

This basic unit is submitted to ecological and chorological variations.

Ecological variations are due mainly to changes of the water quality. They are illustrated by two examples:

- two river systems in the Northem Vosges where a general survey shows longitudinal variations of communities without pollution or assessing different kinds of poltution,

- a pisciculture pollution with observations of species distribution on consecutive stretches.

An Increase of water trophic level leads progressively from an oligotrophic vegetation (marked by Potamogeton polygonifolius, Juncus bulbosus, etc.) to a meso-eutrophic community (characterized by Callitriche obtusangula, Nasturtium officinale, etc.). Four communities are distinguished in Northern Vosges (table 2) and characterize water quality (both pollution and trophic (evel) :

A : Potamogeton polygonifolius, Juncus bulbosus, Sphagnum spp. community, with the following main water-quality parameters : conductivity : $40-80 \mu \mathrm{S}$, phosphates : $<20 \mathrm{ppb} \mathrm{P}$, ammonia : $<20 \mathrm{ppb} \mathrm{N}$;

B : Potamogoton polygonifollus, Ranunculus peltatus, Callitriche hamulata community, $(40-80 \mu S, 20-40 \mathrm{ppb}$ P, ammonia : $\angle 40 \mathrm{ppb} \mathrm{N})$

C: Ranunculus peltatus, Callitriche hamulata, Potamogeton alpinus, Myriophyllum altermiflorum community, $(60-120 \mu \mathrm{S}, 40-120 \mathrm{ppb}$ P, ammonia : $<200 \mathrm{ppb} N)$

D : Callifriche obfusangula and Nasturtium officinale inside C community list,

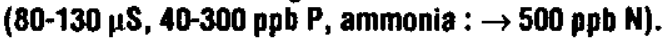

Two vegetation maps show the distribution of these communities (flg. 2). The succession of communtities is related to water quality and fastened sequences are compared to normal ones. Without any pollution, $A$ and $B$ communities cover more than $5 \mathrm{~km}$ along the upper streams; under these conditions, the entire sequence $A$ to $D$ develops to $15 \mathrm{~km}$, while with much anthropic pollution, It may occur within less than $3 \mathrm{~km}$. A heavy pollution leads to the lack of communities as observed with the change from $A$ to $C$ (without any $B$ ) due to a camping-site.

A localized organic pollution by a Brelon pisciculture is responsible for waterquality changes (table 3): ammonia, nitrites, phosphates and conductivity increase. The succession of mineral nitrogen forms is assessed (fig. 3). In the $\mathrm{N}-\mathrm{NH}_{4}+$ and $\mathrm{N}-\mathrm{NO}_{2}-$ peak areas, the eutrophic species increase (Callitriche spp., specially Callitriche oblusangula, Amblysteglum riparium) and the oligotrophic ones decrease (Scapania undulata) (fig. 3). Tissue analysis of Ranunculus penicillatus and of Callitriche spp. does nol show any enrichment In nitrogen in polluted areas: the ecological response is mainly an increase of nitrophilous macrophytes (Callitriche spp.).

The Influence of water quality is more obvious in the Northern Vosges for most of the ollgotrophic communities grow in forested stretches, while in Brittany these conditions are rare and eutrophicalion occurs in all the streams, even in the source areas. Floristicalty, neutrophilous or ubiquitous species are numerous. Some of them indicate either eutrophication or pollution : Amblystegium riparium and perhaps Octodiceras fontanum for bryophyta, 
Callitriche obfusangula, Potamogeton perfoliatus and Sparganium. emersum (long leaves form), for aquatic species, Nasfurfium officinale, Glyceria maxima for helophytes.

Physical parameters also lead to variations. The succession of swift and slow areas leads to changes in the communities. Thus, in Brittany, Oenanthe crocata and Ranunculus peniclliatus are associated on the rifiles, while Nuphar Iutea and Sparganium emersum characterize the pools, even in the upper parts of the streams where there are watermill ponds.

The relationships belween local hydrodynamic features and species distribution are discussed with regards to the stretches around the pisciculture area.

Main chorological variation is an enrichment of atlantic areas with numerous eu-attantic or mediterraneo-atlantic species : Porella pinnata and Amblystegium fiuviatile as bryophyta, Oenanthe crocata, Apium inundatum, Ranunculus omiophyllus as spermaphyta. As there are few or no species restricted to medio-european areas, Atlantic communities are often richer than eastern ones. But some invading specles as Elodea nuttallij are spreading all over the country from North-Eastern France.

Ecological trends of certain species (Sparganium emersum, Ranunculus peltatus, Potamogeton polygonifolius) can change between the Iwo biogeographical areas. So, Sparganium emersum grows in the upper oligotrophic zones in Northern Vosges while it characterizes lower meso-eutrophic zones in Brittany. In Brittany, Ranunculus peltatus can exist in eutrophic areas when the stretches are far from the sea. Potamogelon polygonifolius is very rare in swift streams in Brittany.

Therefore, macrophyles permit, as benthic invertebrates do, to get a river biodiagnosis. They are easily observed and mapped so they can be used for a general survey.

Key-words : acid rivers, macrophyte, bioindicators, water quality, chorology.

\section{INTRODUCTION}

La végétation des cours d'eau acides faiblement minéralisés est assez mal connue en France. Des inventaires floristiques et études phytosociologiques s'avèrent donc nécessaires, et ce d'autant plus rapidement que l'eutrophisation (dans le Massit armoricain comme dans les Vosges du Nord) et l'hyperacidification (dans quelques rivières du Massif vosgien) risquent de faire disparaître certaines phytocénoses oligotrophes. Les cormophytes seront envisagés, et seuls seront cités les travaux concernant les rivières acides.

De nombreuses questions méthodologiques concernant les relevés de végétation aquatique ont été soulevées par HOLMES et WHITTON (1977), HASLAM (1978), HAURY (1982 et 1985), WIEGLEB (1983a); une mise au point méthodologique est actuellement en cours (LEGLIZE et al, 1990). Seuls les groupements et espèces qui apparaissent franchement aquatiques et subaquatiques sont pris en considération dans cet article. 


\section{1- MÉTHOdES ET LIEUX d'ÉTUdE}

\subsection{Méthodologies}

\subsubsection{Les relevés de la végétation}

Les relevés de végétation ont été effectués selon les règles suivantes:

- un inventaire floristique précis (jusqu'à l'espèce) de l'ensemble des secteurs d'étude, comme le préconisent HOLMES et WHITTON (1977),

- une longueur minimale échantillonnée de 50 m (HAURY, 1982; WIEGLEB, 1983a), voire davantage lorsque la végétation est disséminée, ceci pour atteindre l'épuisement floristique,

- une distinction entre les biotopes aquatiques et les zones marginales et ripicoles, notamment pour la définition des groupements végétaux.

L'échantillonnage stratifié a été retenu pour l'étude des grands bassinsversants. Pour l'examen des variations longitudinales des cours d'eau, une prospection systématique a été pratiquée (HAURY, 1988a; MULLER, 1990), avec un éventuel découpage en unités consécutives de 50 m (HAURY, 1985 et 1988a).

Les indications sur l'écologie des espèces ont été obtenues par des observations qualitatives, le tracé des profils écologiques (HAURY, 1985; 1989 et 1990) et des renseignements bibliographiques (AUGIER, 1966; HASLAM et al., 1975 ; HASLAM, 1978 et 1987 ; SMITH, 1980 ; MERIAUX, 1982; HOLMES, 1983 ; WIEGLEB, 1983a, 1983b et 1988). Les nomenclatures de référence sont celles de CORLEY et al. (1982) pour les mousses, de GROLLE (1983) pour les hépatiques, et de TUTIN et al. (1964 à 1980) pour les végétaux vasculaires.

\subsubsection{Les relevés mésologiques et les analyses d'eau}

La mésologie physique a surtout été étudiée en Bretagne, compte tenu des importants aménagements des cours d'eau qui y sont réalisés et définissent une différenciation des faciès d'écoulement, notamment par la création des * unités inter-barrages * (HAURY, 1982). Les méthodes d'étude ont été précisées par ailleurs (HAURY, 1985 et 1989). Les cours d'eau prospectés varient d'un ordre de drainage de 1 à 5 dans le Massif armoricain, pour une largeur comprise entre 0,4 et $16 \mathrm{~m}$ et des profondeurs extrêmes de $0,1 \mathrm{~m}$ à 2,3 m. Dans les Vosges du Nord, ces grandeurs sont : 1 à 4 pour l'ordre de drainage, 0,4 à $7 \mathrm{~m}$ pour la largeur et 0,1 à $1,5 \mathrm{~m}$ pour la profondeur.

Deux groupes de méthodologies ont été appliquées aux échantillons d'eau récoltés :

- analyses en laboratoire (Laboratoire de Génie sanitaire de l'Ecole Nationale de la Santé Publique à Rennes pour la pollution par la pisciculture de Pont-Calleck, et Laboratoire d'Ecologie de l'Université de Metz pour l'ensemble des cours d'eau vosgiens) selon les méthodes normalisées; les résultats obtenus pour la Bretagne ont servi de référence pour l'approche suivante :

- analyse par spectrocolorimétrie après étalonnage sur solutions-témoins pour les formes de l'azote (Massif armoricain). La précision de ces mesures est d'au moins $10 \%$. 
En outre, la température, le pH et la conductivité sont mesurés sur le terrain par des pHmètres et conductimètres portables.

\subsection{Les lieux d'étude}

Les régions naturelles et les réseaux étudiés sont représentés sur les figures 1 .

Les principales rivières étudiées dans le Massif armoricain sont situées sur des roches acides : granites, grès, schistes ou gneiss. Ce sont de l'Ouest vers I'Est, l'Elorn, le Scorff (HAURY, 1982, 1985 et 1988a), le Trieux (HAURY, 1988b, 1989 et 1990), l'Aff amont et la Flume, et l'Oir ; en outre, des ruisseaux des Monts d'Arrée, du Bassin de Rennes, de Normandie et de la Mayenne armoricaine ont fait l'objet de relevés plus localisés.

La pisciculture de Pont-Calleck est implantée à mi-cours du Scorff (fig. 1b), à l'aval d'un affluent important (le Bras de Pont-Calleck); un barrage permet l'alimentation en eau de la pisciculture et crée donc un "profond" amont (1,5 $\mathrm{m}$ au maximum). Les exutoires de chaque bassin sont indépendants et les rejets se font directement à la rivière. A l'aval, une digue de moulin crée une autre zone profonde $(1,5 \mathrm{~m}$ à $2 \mathrm{~m})$. Les tronçons étudiés sont :

$-300 \mathrm{~m}$ sur l'affluent (prélèvement $\mathrm{C}$ ),

- $700 \mathrm{~m}$ sur le Scorff à l'amont du barrage (prélèvement $M$ ),

- l'ensemble de l'unité interbarrage sur le Scortf, soit $3,4 \mathrm{~km}$, les prélèvements se faisant à $100 \mathrm{~m}$ du dernier rejet $(P)$ et dans la zone de récupération à $2 \mathrm{~km}(\mathrm{~V})$ du point $P$.

Dans les Vosges du Nord, la plupart des cours d'eau ayant leur source au niveau du grès vosgien ont été prospectés. Ce sont essentiellement, du nord au sud, le Steinbach, le Schwarzbach, le Falkensteinbach, la Zinsel du Nord, le Rothbach et la Moder, tous situés dans le bassin-versant du Rhin.

Les deux rivières vosgiennes particulièrement étudiées sont le Schwarzbach et le Rothbach.

\section{2 - LA VÉGÉTATION DES RIVIÈRES ACIDES ET SES PRINCIPALES VARIATIONS}

\subsection{Résultats dans le Massif armoricain et les Vosges du Nord}

Les groupements macrophytiques constituent des séquences amont-aval qui traduisent les grandes zones écologiques de ILLIES et BOTOSANEANU (1963) (tableau 1).

\subsubsection{Associations fontinales et des petits ruisselets}

Dans les têtes de bassin-versant (le crénon et l'hypocrénon), les groupements fontinaux des milieux tourbeux acides (oligotrophes humiques) 


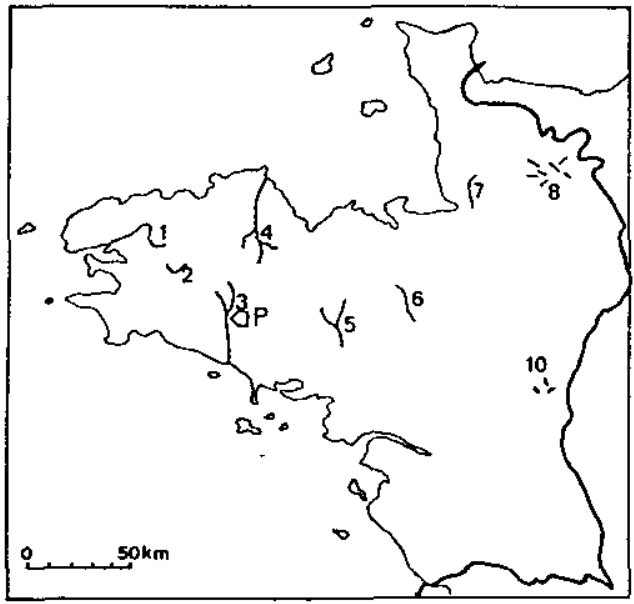

b)

- LIMITE OU MASSIF ARMORICAIM (MASSIF ARMORICAIN LIMIT)

COTES (COAST)

RIVIERES ETUOIEES (STUDY RTVERS)

P PISCICULTURE

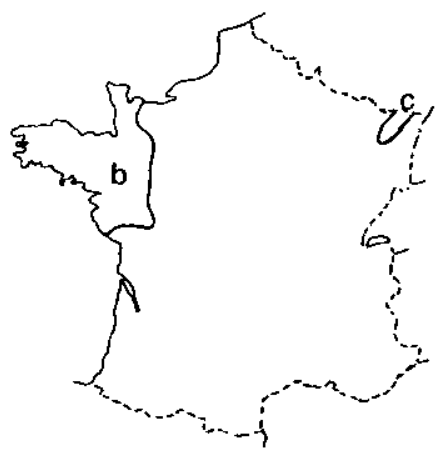

a)

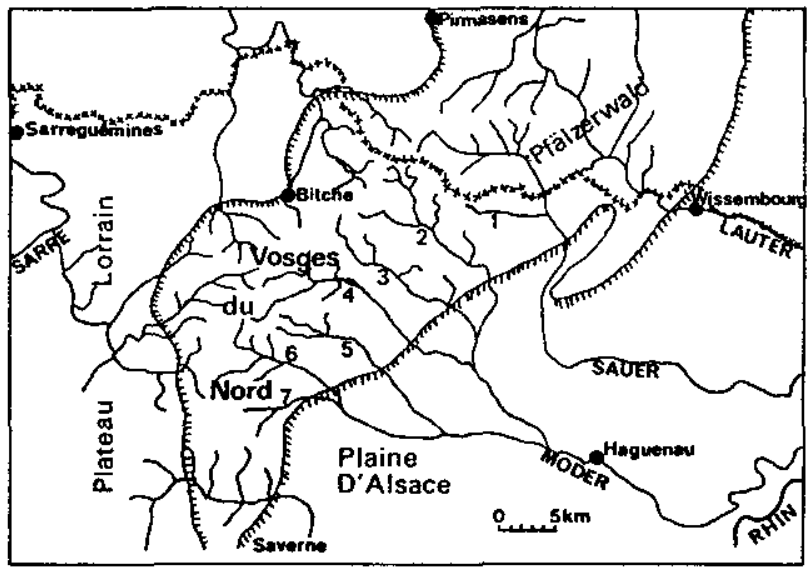

c)

COURS DEAU (RIVER)

ME: FRONTIERE (BORDER)

LIMITESDES VOSGES

- VILLE (TOWN)

(NORTHERN VOSGES LIMITS)

Figures 1 Lieux d'étude

a) Régions naturelles

b) Rivières du Massif armoricain

1: Elom ; 2 : Ruisseaux des Monts d'Arrée ; 3 : Scorff ; 4 : Trieux ; 5 : Aff amont ; 6 : Vaunoise ;

7: Fume; 8 : Oir ; 9 : Ruisseaux nomands ; 10 : Ruisseaux mayennais.

c) Rivières des Vosges du Nord

1: Steinbach ; 2 : Schwarzbach ; 3 : Falkensteinbach ; $4:$ Zinsel du Nord ; 5 : Rothbach ;

6: Moder; 7 : Mittelbach.

\section{Study areas}

a) Natural Entities

b) Rivers of the Armorican Massif

c) Aivers of the Northern Vosges 
Tableau 1 Répartition des principaux macrophytes dans les cours d'eau du Massif armoricain et des Vosges du Nord. Zonation longitudinale, variations écologiques et chorologiques.

Table 1 Distribution of main macrophytes in rivers of the Armorican Massif and of the Northern Vosges. Longitudinal zonation, ecological and chorological vanations.

\begin{tabular}{|c|c|c|}
\hline Biotope/Zones Crénon & Rhitron & Potamon \\
\hline $\begin{array}{l}\text { Rives ombragees } \\
\text { Cardamines pl. sp. } \\
\text { Pellia epiptylla } \\
\text { Cirysosplenium oppositiolium }\end{array}$ & $\begin{array}{l}\text { Rhynchostegium riparioïdes } \\
\text { Fontinalis antipyretica } \\
\text { Fontinalis squamosa* } \\
\text { (Amblystegium fluviatile) } \\
\text { Porella pinnata } \\
\text { Chiloscyphus polyanthos } \\
\text { Fissidens pusilus } \\
\text { Amblystegium riparium E } \\
\text { Riccardia chamaedryfolia } \\
\text { Conocephalum coricum } \\
\text { Lunularia cnuciata E } \\
\text { Cinclidotus sp. } \\
\text { Thamnobryum alopecunum }\end{array}$ & (Octodiceras fontanum) ME \\
\hline $\begin{array}{l}\text { Lh éclains } \\
\text { Potamogeton polygonifolius *0 } \\
\text { Juncus bulbosus *0 } \\
\text { Sphagnum } \mathrm{Sp} \text { * } 0 \mathrm{t} \\
\text { Glyceria fluitans } \\
\text { Scimpus fluitans } \\
\text { Ranunculus omiophyllus *0 } \\
\text { (Ranunculus hederaceus) ME } \\
\text { Montia fontana } \\
\text { Callitriche stagnalis } \mathrm{M} \\
\text { Nasturtium officinale } E\end{array}$ & $\begin{array}{l}\text { (Ranunculus penicillatus) } \\
\text { Ranunculus pettatus Dom. } \\
\text { Callitriche hamulata* } \\
\text { Callitriche platycarpa } \\
\text { Myriophylum altemiftonum* } \\
\text { Oenanthe fluviatile ME } \\
\text { Oenanthe crocats } \\
\text { Luronium natans } M \\
\text { Apium inundatum } M \\
\text { Potamogelon polygonifolius o } \\
\text { Potamogeton berchioldii } \\
\text { Callitriche obtusangula E } \\
\text { (Nasturtium oflicinale) ME } \\
\text { Potamogeton perfoliafus ME }\end{array}$ & $\begin{array}{l}\text { Sparganium emersum long.ME } \\
\text { Nuphar lutea } M E \\
\text { Potamogeton crispus } E \\
\text { Elodea canadensis } \\
\text { Elodea nuttallii E } \\
\text { Nymphaea alba M } \\
\text { Potamogeton panomitanus } \\
\text { Lemna minor }\end{array}$ \\
\hline $\begin{array}{l}\text { Rives éclaintes } \\
\text { Sparganium erectum } \\
\text { Carex paniculata } \\
\text { Carex rostrata * }\end{array}$ & Pthalaris anundinacea & $\begin{array}{l}\text { Phalaris arundinacea } \\
\text { Typha latifolia }\end{array}$ \\
\hline
\end{tabular}

Lógendo Les espèces communes aux deux régions et dans la même zone figurent en caractères droits, celles retrouvées seulement en Bretagne figurent en italiques, celles exclusives des Vosges en gras. Les parenthèses indiquent une faible fréquence. Les optimas sont seuls indiqués. Les lettres se rapportent aux prétérences trophiques des espcèes : 0 pour oligotrophe et 0 t pour oligotrophe tourbeux, $M$ pour mésotrophe, E pour eutrophe. L'astérisque * indique les espèces majeures du fond commun acidophile.

Legend Species belonging to both regions and in the same zone are in normal prints. Bitton ones are in italics, and vosgian ones in thick prints. Brackets means a scarcity in the rivers. Only optima are presented. Characters indicate trophic preferences : O for oligotrophic ones, Ot for peaty oligotrophic, $M$ for mesotrophic and $E$ for eutrophic preferences. Asterisk - indicates main acid species. 
et oligotrophes sont dominés par Potamogeton polygonifolius accompagné ou non par des Sphaignes, par Glyceria fluitans et Juncus bulbosus, et en outre en milieu océanique, par Scirpus fluitans. Ces communautés sont assez tolérantes à l'ombrage. G. fluitans est présent dans toutes les régions.

En milieu oligo(méso)trophe non tourbeux et bien éclairé, on trouve les communautés à Montia fontana, ainsi que celles à petites renoncules (Ranunculus omiophyllus, Ranunculus hederaceus, ...) développées exclusivement dans les zones océaniques.

En zone ombragée, le crénon est marqué par les communautés à Scapania undulata, cette espèce pouvant être associée à d'autres bryophytes, comme des Sphaignes ou Chiloscyphus pallescens.

Dans les petits ruisseaux éclairés (hypocrénon et épirhitron), différentes communautés d'Oenanthides sont représentées par Apium nodiflorum, Oenanthe crocata (en zone océanique). Nasturtium officinale est fréquente en milieu enrichi dans ces biotopes en Bretagne. G. fluitans y est souvent abondante tant dans le lit qu'à proximité des berges.

Dans les fossés et ruisseaux lents, on rencontre en outre Callitriche stagnalis (exclusive de ces milieux et correspondant à des conditions trophiques assez élevées) et/ou Callitriche platycarpa qui trouve son optimum dans la zone suivante.

Sparganium erectum, mais aussi la forme terrestre de G. fluitans en milieu oligotrophe, Carex paniculata et Carex rostrata en zone tourbeuse forment des communautés subaquatiques qui tendent à envahir les ruisselets éclairés.

\subsubsection{Le titron}

La flore bryophytique est plus développée dans le Massif armoricain, les ruisseaux des Vosges du Nord ayant généralement un fond sableux. Elle est dominée par des Fontinelles, Fontinalis antipyretica et Fontinalis squamosa, par Fissidens pusillus, Rhynchostegium riparioïdes et Riccardia chamaedryfolia. On y trouve encore S. undulata souvent associée à Chiloscyphus polyanthos. Amblystegium fluviatile et surtout Porella pinnata sont des différentielles atlantiques ou subatlantiques. Amblystegium riparium indique une dégradation de la qualité de l'eau. Parmi les espèces ripicoles sciaphiles, on trouve Thamnobryum alopecurum et Conocephalum conicum. Lunularia cruciata existe en conditions enrichies en Bretagne.

Dans les rivières et ruisseaux assez importants, les groupements phanérogamiques sont caractérisés par Myriophyllum alterniflorum et Callitriche hamulata, $C$. platycarpa, $P$. alpinus et des Renoncules qui sont souvent très abondantes. Callitriche obtusangula et $N$. offininale (ainsi que Potamogeton perfoliatus et parfois Zannichellia palustris en Bretagne) indiquent une dégradation des conditions de milieu. C'est au sein de ces communautés que les différences chorologiques entre le Massif armoricain et les Vosges du Nord sont les plus nettes :

- Ce ne sont pas les mêmes espèces de renoncules qu'on y rencontre, Ranunculus penicillatus var. penicillatus est surtout présente en milieu océanique, alors que Ranunculus peltatus est dominante en milieu plus continental (GEHU et MERIAUX, 1983), bien que présente dans certaines rivières 
de Bretagne (DES ABBAYES et al., 1971 ; HAURY, 1988b ; ...). En Bretagne, dans les zones calmes, à l'aval d'étangs eutrophes, on trouve en outre quelques pieds de Ranunculus aquatilis.

- $P$. polygonifolius est assez abondant dans les rivières moyennes des Vosges du Nord et rare dans le Massif armoricain (Yeun Ellez dans les Monts d'Arrée et Elorn) dans ces mêmes conditions.

- Oenanthe fluviatilis, présent en quelques stations des Vosges du Nord, est absent des rivières acides de Bretagne, et Potamogeton berchtoldii doit y être recherché, l'espèce de petit Potamot la plus fréquente semblant être Potamogeton panormitanus.

- Les cours d'eau armoricains possèdent des espèces atlantiques ou subatlantiques comme $O$. crocata, Apium inundatum et Luronium natans qui manquent dans les régions continentales comme les Vosges du Nord.

En bordure de ces cours d'eau, se développent des roselières à Phalaris arundinacea. Dans les Vosges du Nord, y apparaissent également Glyceria maxima et Berula erecta; dans le Massif armoricain, G. maxima correspond en général à des eaux enrichies (Canut Nord, Vilaine) et $B$. erecta se trouve notamment à l'aval des calcaires normands (Orne armoricaine).

\subsubsection{Le potamon}

Les bryocénoses exclusives du potamon n'existent pas. Une espèce est à citer en Bretagne où elle est fréquente dans cette zone des cours d'eau acides à neutres : Octodiceras fontanum.

Les groupements héliophiles sont dominés par Sparganium emersum (forme à feuilles longues), Potamogeton crispus, Elodea canadensis, avec un tapis flottant de lentilles d'eau, Lemna minor, dans les endroits calmes. Nymphaea alba, espèce mésoeutrophe, et Potamogeton natans sont rares, mais existent en cours d'eau surtout dans les retenues ou à l'aval d'étangs, étant nettement inféodés aux eaux stagnantes. Trois espèces ont une distribution différente entre le Massif armoricain et les Vosges du Nord:

- P. panormitanus, assez fréquent dans le potamon de Bretagne, ne se trouve pas dans les Vosges du Nord,

- le Nénuphar jaune, Nuphar lutea, espèce la plus caractéristique des * profonds * dans l'Ouest, est absent des Vosges du Nord même en étang.

- Elodea nuttallii, espèce adventice d'introduction récente, n'a pas encore été observée dans le Massif armoricain.

Les communautés ripicoles sont encore dominées par $P$. arundinacea, parfois accompagné de Typha latifolia.

\subsection{Les données bibliographiques}

L'approche floristique par réseau hydrographique a été développée précocement en Angleterre (BUTCHER, 1933) puis dans l'ensemble de l'Europe (KOHLER et al., 1971 ; KOHLER, 1975 ; FABRI et LECLERCQ, 1977 ; HOLMES et WHITTON, 1977 ; HASLAM, 1978 ; WOLFF, 1987 ; HASLAM et MOLITOR, 1988). 
La description des groupements végétaux acidoclines par la méthode sigmatiste n'a pas été abordé Outre-Manche; en revanche, les travaux sur le continent sont nombreux (DEN HARTOG et SEGAL, 1964 ; WIEGLEB, 1983b ; DETHIOUX et NOIRFALISE, 1985). Une synthèse pour l'Europe médioeuropéenne a été établie par OBERDORFER (1977). Pour les bryophytes, les travaux germanophones font autorité (PHILIPPI, 1956 ; VON HUBSCHMANN, 1957 ; MARSTALLER, $1987 ; \ldots$. .

HOLMES (1983) a défini 56 types floristiques dans les rivières anglaises et HASLAM (1987) a effectué un survol des plantes de rivières en Europe de I'Ouest, selon une méthode de diagnose rapide qui exclut toutes les difficultés taxonomiques.

Les observations réalisées en France concordent assez bien avec les typologies de HOLMES (1983) et de HASLAM (1978 et 1987). Parmi les especes additionnelles par rapport au tableau 1 citées dans la bibliographie, on distingue des espèces montagnardes comme Scapania paludosa, Schistidium alpicola, certains macrophytes rares (au moins en cours d'eau) comme Eleocharis acicularis en Irlande, Hydrocharis morsus-ranae en Belgique et au Luxembourg, Sparganium minimum (HASLAM, 1987), des espèces qui ont pu être confondues dans les relevés (Amblystegium tenax et A. fluviatile), des taxons plutôt subaquatiques (Schistidium alpicola, Racomitrium aciculare, ...).

\subsection{L'ensemble acidophile et ses différentlelles écologiques et chorologiques}

En conclusion, le fond commun acidophile est marqué par quelques espèces bien représentées dans les deux régions: Sphaignes, Scapania undulata, ... pour les bryophytes, et $P$. polygonifolius, $J$. bulbosus, $C$. hamulata, $P$. alpinus, $M$. alterniflorum, ..., pour les phanérogames.

Les neutrophiles ou indifférentes sont nombreuses. Certaines d'entre elles sont indicatrices de trophie plus élevée dans les cours d'eau acides. Ce sont $A$. riparium et peut-être $O$. fontanum, pour les bryophytes, $C$. obtusangula, Potamogeton perfoliatus et $S$. emersum (forme à feuilles longues), et, dans une moindre mesure, $P$. crispus, $E$. canadensis et $E$. nuttallii, pour les hydrophytes, $N$. officinale, $G$. maxima et, moins nettement, $B$. erecta pour les hélophytes.

Les espèces atlantiques et subatlantiques sont abondantes: Porella pinnata, A. fluviatile, ... pour les bryophytes, et $A$. inundatum, $O$. crocata, R. omiophyllus, ... pour les phanérogames.

Le cortège spécifiquement médio-européen est quasi-inexistant; ceci implique qu'a priori, les phytocénoses atlantiques seront plus diversifiées à niveau trophique et zone écologique équivalents. Toutefois, $R$. peltatus semble plus développée en milieu continental et $R$. penicillatus var. penicillatus en zone océanique.

Certaines adventices récentes, comme Elodea nuttallii, sont encore assez rares en France, quoiqu'en expansion à partir du Nord-Est du pays. 


\section{3 - EXEMPLES DE VARIATIONS ÉCOLOGIQUES}

Les listes générales précédentes masquent les variations qualitatives (apparition ou disparition d'espèces) ou quantitatives (modifications des recouvrements spécifiques) au sein des réseaux hydrographiques.

\subsection{La séquence d'eutrophisation des Vosges du Nord}

L'étude phytosociologique de la végétation des eaux courantes des Vosges du Nord a permis d'individualiser quatre groupements végétaux (symbolisés par les lettres A, B, C et D), se succédant normalement d'amont en aval et correspondant à une augmentation du niveau trophique (MULLER, 1990) (tableau 2).

Tableau 2 Distribution des principales espèces dans les quatre groupements de la séquence des Vosges du Nord - Caractéristiques physico-chimiques des eaux.

Table 2 Distribution of main species inside the for communities of Northern Vosges sequence - Water quality features.

\begin{tabular}{|c|c|c|c|c|}
\hline Groupements & A & B & C & D \\
\hline Sphagnum sect subsecunda & t+ & - & - & - \\
\hline Potamogeton polygonitolius & $H$ & +t & - & - \\
\hline Juncus bulbosis & t+ & t+ & - & - \\
\hline Carex rostrata & $H$ & t+ & - & - \\
\hline Glycería fluttans & H & ++ & $(+)$ & - \\
\hline Callitriche hamudata & - & ++ & ++ & ++ \\
\hline Callitriche platycarpa & - & t+ & + & H \\
\hline Ranunculus peltatus & - & t+ & H & H \\
\hline Spargarium emersum & - & t+ & H & H \\
\hline Fontinalis antipyretica & - & + & H & H \\
\hline Phalaris anundinacea & - & $(t)$ & $H$ & H \\
\hline Potamogeton berchtoldii & - & - & H & ++ \\
\hline Elodea nuttallii & - & - & H+ & H \\
\hline Elodea canadensis & - & - & t+ & $?$ \\
\hline Oenanthe fluviatilis & - & - & + & + \\
\hline Potamogeton alpinus & - & - & + & $?$ \\
\hline Myriophylum altemifilonm & - & - & + & $?$ \\
\hline Nasturtium officinale & - & - & $(+)$ & t+ \\
\hline Callitriche obtusangula & - & - & - & $H$ \\
\hline $\begin{array}{c}\text { Caractéristiques } \\
\text { physico-chimiques }\end{array}$ & $A$ & B & $\mathbf{C}$ & D \\
\hline $\begin{array}{l}\text { pH } \\
\text { Conductivité }(\mu S)\end{array}$ & $\begin{array}{l}5,5-6,5 \\
40-80\end{array}$ & $\begin{array}{c}5,5-6,5 \\
40-80\end{array}$ & $\begin{array}{c}6-7 \\
60-120\end{array}$ & $\begin{array}{l}6,5-7,5 \\
80-130\end{array}$ \\
\hline $\begin{array}{l}\text { Titre Alcalimétrique } \\
\text { Complet (T.A.C. of) }\end{array}$ & $0,3-1$ & $0,4-1,5$ & $1-2$ & $2-3$ \\
\hline $\begin{array}{l}\mathrm{PO}_{4}^{3-}(\mathrm{ppb} \mathrm{P}) \\
\mathrm{NH}_{4}^{+}(\mathrm{ppb} \mathrm{N}) \\
\mathrm{NO}_{3}^{-}(\mathrm{ppm} \mathrm{N})\end{array}$ & $\begin{array}{l}<20 \\
<20\end{array}$ & $\begin{array}{l}20-40 \\
<40 \\
\text { Variable }\end{array}$ & $\begin{array}{l}40-120 \\
<200 \\
\text { rs }<1)\end{array}$ & $\begin{array}{l}40-300 \\
\rightarrow 500\end{array}$ \\
\hline
\end{tabular}

Légende :

- : absence $;(+),+,++$ : présence croissante
Legend:

$-:$ absent $:(+), t_{1}++$ : more and more present 
- Le groupement A est marqué par l'abondance de $P$. polygonifolius, qui s'y développe en compagnie de $J$. bulbosus et souvent de Sphaignes ;

- le groupement $\mathrm{B}$ est différencié par la persistance de $P$. polygonifolius associé à $R$. peltatus et à des Callitriches, $C$. platycarpa et $C$. hamulata, qui manquaient en $A$;

- ensuite, le groupement $C$ s'individualise par la disparition de $P$. polygonifolius et la coexistence de $C$. platycarpa et $C$. hamulata avec R. peltatus et les Elodées ( $E$. canadensis et $E$. nuttallii), ainsi que quelques epèces plus rares dans cette région, mais très typiques de ce groupement: $M$. alterniflorum et $P$. alpinus;

- enfin le groupement D correspond à l'apparition, parfois massive, de C. obtusangula et de $N$. officinale dans l'ensemble spécifique C.

Ces groupements forment normalement, de $\mathrm{A}$ à $\mathrm{D}$, des zonations longitudinales amont-aval, correspondant à une augmentation du débit de ces rivières et à un accroissement de la minéralisation et de la trophie, ce qui est traduit par les chiffres $d u \mathrm{pH}$, de la conductivité et du titre alcalimétrique complet (tableau 2).

Une telle biotypologie de la qualité de l'eau à partir des groupements macrophytiques permet aisément une représentation cartographique, comme l'illustrent les cartes des niveaux trophiques croissants de deux cours d'eau vosgiens élaborés à partir des groupements végétaux (fig. 2). Elles mettent en évidence :

- le rôle joué par des villages et autres installations humaines (camping, étangs de pisciculture) comme sources d'éléments nutritifs, dans l'évolution de ces deux séquences; des séquences accélérées peuvent se traduire par des sauts de certains stades (par exemple $A \rightarrow C$ à l'aval d'un terrain de camping),

- l'existence de secteurs de rivière pratiquement inaltérés (groupements $A$ et B) sur des distances de plus de $5 \mathrm{~km}$ en l'absence de perturbation anthropique,

- alors que pour d'autre ruisseaux subissant des apports nutritifs importants, on passe en $3 \mathrm{~km}$ du groupement $A$ au groupement D qui traduit des teneurs importantes en azote ammoniacal et en phosphates.

\subsection{Les effets d'une pollution organique sur les phytocénoses d'une unité inter-barrages (fig. 3)}

Les rejets de la pisciculture de Pont-Calleck se traduisent par une modification sensible de la qualité de l'eau. Les caractéristiques des eaux de l'affluent, de l'amont, de l'aval immédiat $(150 \mathrm{~m})$ des derniers rejets et de la zone de récupération $(2,1 \mathrm{~km}$ à l'aval des derniers rejets) sont résumées dans le tableau 3 qui correspond à un cycle annuel. L'altération la plus marquante est la succession des pics des formes de l'azote, les échelles étant modifiées pour mettre en évidence ce phénomène (fig. 3a).

Plusieurs phénomènes peuvent être illustrés par l'examen des recouvrements des macrophytes, tant en zone marginale qu'en plein courant. On observe à laval des rejets :

- une régression de $S$. undulata, espèce polluo-sensible (fig. $3 b$ ),

- un développement important de $A$. riparium (fig. $3 c$ ), 

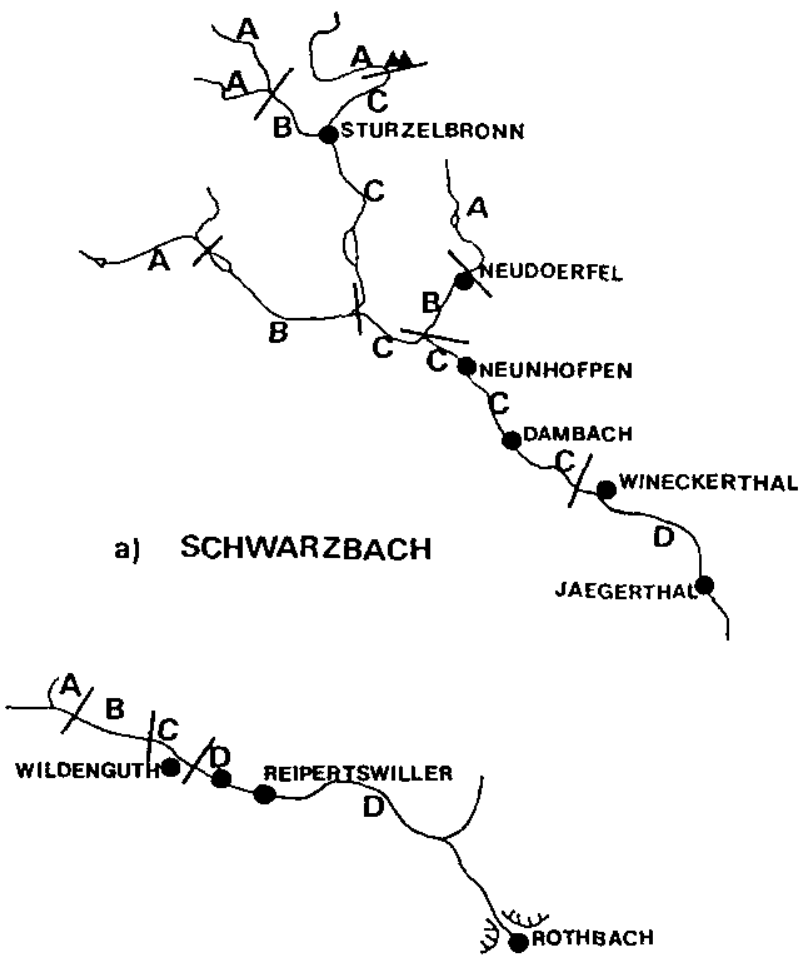

b) ROTHBACH

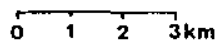

4. CAMPING

- village (VILlage)

A,B,C,D GRoupements (PLANt COMmunities)

Figures 2 Séquences longitudinales des groupements végétaux dans deux rivières des Vosges du Nord.

a) Séquence normale : $A \rightarrow D=15 \mathrm{~km}$,

b) Séquence accélérée : $A \rightarrow D=3 \mathrm{~km}$.

Longitudinal zonation of plants communities in two Northem Vosges rivers.

a) Normal sequence: $A \rightarrow D=15 \mathrm{~km}$,

b) Fastened sequence: $A \rightarrow D=3 \mathrm{~km}$.

- une progression des Callitriches (C. platycarpa et C. obtusangula), notamment de la seconde (fig. 3d),

- aucune différence significative pour $F$. antipyretica (fig. $3 e$ ).

D'autres espèces montrent des différences de recouvrement: Amblystegium riparium, S. emersum (la forme rhéophile), E. canadensis et $N$. officinale semblent progresser à l'aval des rejets, de même que les lentilles d'eau, Lemna minor. En revanche, Apium inundatum et $M$. alterniflorum régressent dans la zone d'enrichissement en azote ammoniacal et nitreux. 


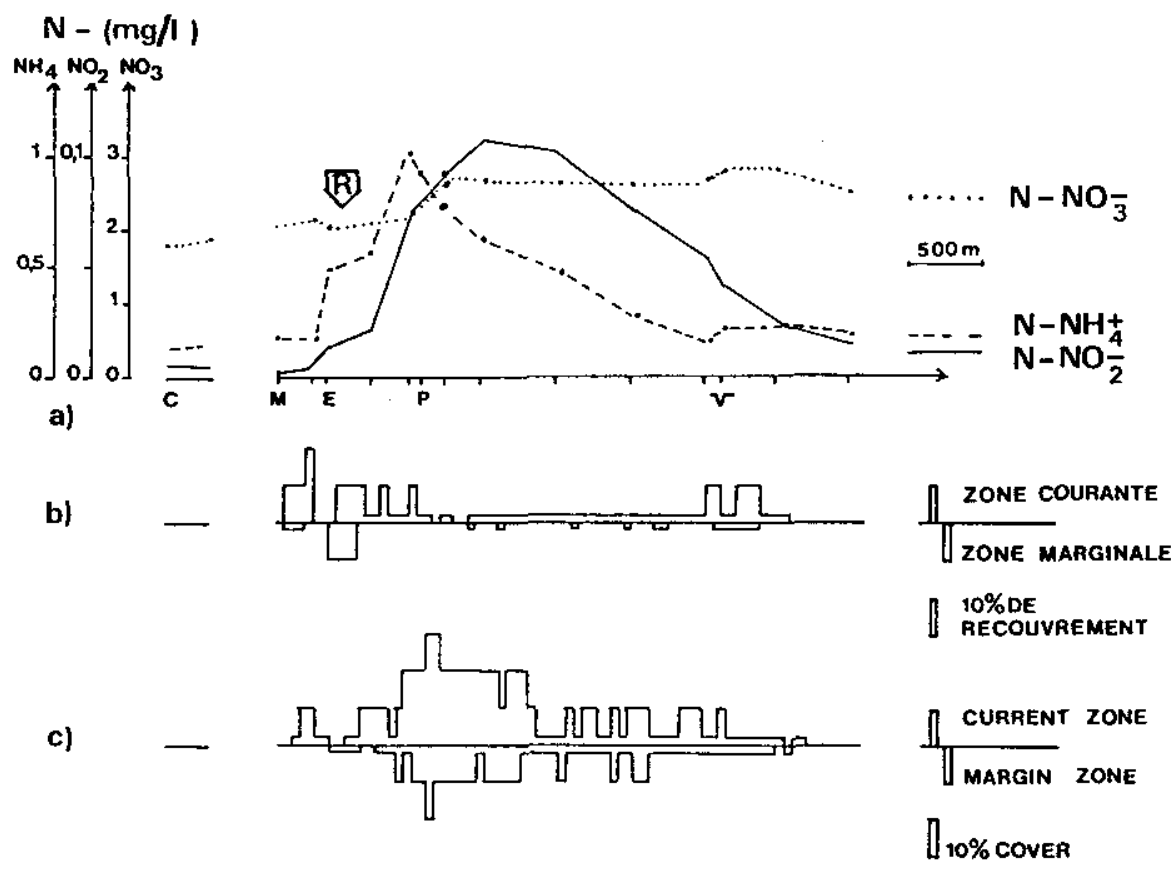

d)

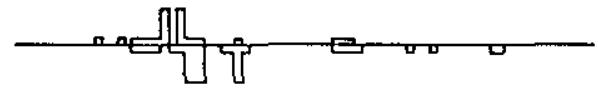

e)
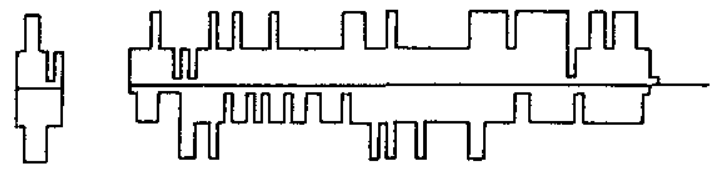

f)
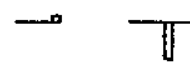

g)

Figures 3 Pollution par la pisciculture de Pont-Calleck et recouvrements végétaux. a) Formes de l'azote - R : zone des rejets ; C, P, M, V: points de prélèvements; $E$ : un effluent de bassin.

b) Scapania undulata ; c) Amblystegium riparium ; d) Callitriche obtusangula (forme avec rosettes); e) Fontinalis antipyretica; f) Nuphar lutea ; g) Typha latifolia.

Pollution by Pont-Callek pisciculture and macrophytes cover.

a) Nitrogen forms - $R$ : effluent area; $C, P, M, V$ : water sampling points ; $E$ : one tank effluent.

b) Scapania undulata; c) Amblystegium riparium; d) Callitriche obtusangula; e) Fontinalis antipyretica; f) Nuphar lutea ; g) Typha latifolia. 
Tableau 3 Quelques paramètres de la physico-chimie de l'eau au "Nceud" de Pont-Calieck (Analyses E.N.S.P.) - Valeurs moyennes sur un cycle annuel (et extrêmes observés).

Table 3 Some water quality features arount Pont-Calleck (Analysis by E.N.S.P.)) - Average data over a year (and extrema).

\begin{tabular}{|c|c|c|c|c|c|}
\hline Paramètres & Secteurs & C & M & $\mathbf{P}$ & $\mathbf{v}$ \\
\hline $\mathrm{pH}$ & & 6,67 & 6,74 & 6,64 & 6,64 \\
\hline Conductivité $(\mu S)$ & & 141 & 134 & 144 & 139 \\
\hline $\mathrm{NO}_{3}^{-}\left(\mathrm{mg} \cdot \mathrm{r}^{-1}\right)$ & & 12,7 & $\begin{array}{c}15,2 \\
(23,5)\end{array}$ & 12,9 & 14,8 \\
\hline $\mathrm{NO}_{2}-(\mathrm{mg} \cdot \mid-1)$ & & 0,05 & 0,02 & 0,14 & 0,14 \\
\hline $\mathrm{NH}_{4}+\left(m g-r^{-1}\right)$ & & 0,12 & 0,10 & 0,41 & 0,17 \\
\hline $\mathrm{PO}_{4}^{3-}\left(\mathrm{mg} \cdot r^{-1}\right)$ & & 0,13 & 0,14 & 0,47 & 0,41 \\
\hline
\end{tabular}

Légende:

$C$ : Affiluent de Pont-Calleck; $M$ : Amont ; $P$ : $100 \mathrm{~m}$ aval de

Legend:

la Pisciculture ; $V: 2,5 \mathrm{~km}$ aval de la Pisciculture.

$C:$ affluent $: M:$ Upstream; $P: 100 \mathrm{~m}$ downstream from the pisciculture; $V: 2,5 \mathrm{~km}$ downstram from the pisciculture.

Des analyses de teneurs en azote total (méthode Kjeldhal avec préréduction à l'acide salicylique) effectuées sur $R$. penicillatus, $C$. hamulata et $C$. obtusangula n'ont montré aucune variation quantitative significative amontaval des teneurs en azote des tissus, mais de fortes différences interspécifiques, les callitriches ayant des teneurs environ deux fois plus importantes que les renoncules. L'influence de la pollution azotée est donc plutôt une extension des espèces nitrophiles qu'un enrichissement tissulaire.

Les conditions hydrodynamiques entraînent des modifications localisées au sein des phytocénoses.

- La zone profonde et lente de la prise d'eau de la pisciculture est marquée par la présence de Nuphar lutea (fig. 3f) et de la forme à feuilles longues de Sparganium emersum. Le bief de l'aval de la zone étudiée, régulièrement curé et profond $(1,8$ à $2 \mathrm{~m})$ ne possède pas d'hydrophyte spécifique, mais seulement Typha latifolia (fig. $3 g$ ) en zone marginale. Les barrages correspondent donc à des taciès appartenant au potamon, dans des zones écologiques qui appartiennent naturellement au rhitron.

- Les radiers et hauts-fonds constituent des biotopes particuliers marqués par l'abondance de $R$. penicicillatus et surtout de $O$. crocata (HAURY, 1985).

La hiérarchie des facteurs écologiques dans l'organisation des phytocénoses a été précisée par des analyses en composantes principales et des analyses factorielles des correspondances (HAURY, 1985). Celles-ci font apparaître, pour les groupements du Massif armoricain, la primauté des facteurs morphométriques sur les paramètres de la qualité de l'eau pour les macrophytes. 


\section{4 - DISCUSSION - CONCLUSION}

\subsection{Les macrophytes comme bioindicateurs}

\subsubsection{Ecologie et distribution spécifiques}

La comparaison des phytocénoses du Massif armoricain et des Vosges du Nord fait bien apparaître des convergences sur la distribution de nombreuses espèces. Ainsi, les groupements observés à l'amont de la pisciculture de PontCalleck seraient à rapprocher du type $C$ de la typologie élaborée dans les Vosges du Nord (MULLER, 1990), et ceux de l'aval plutôt du type D.

Toutefois des différences d'écologie spécifique apparaissent entre l'Est et l'Ouest de la France. Ainsi par exemple:

- en Bretagne, $R$. peltatus semble surtout présente en cours d'eau enrichi (Trieux) voire eutrophisé (avec Spirodela polyrhiza et de $C$. obtusangula dans le Bassin rennais), son amplitude trophique étant plus large dans les Vosges du Nord où il apparaît dès le stade B (oligomésotrophe) ;

- Sparganium emersum montre une double tendance écologique : on le trouve dans les zones oligomésotrophes des ruisseaux vosgiens, en zone assez courante (avec une forme a feuilles courtes et peu épaisses), et en milieu nettement eutrophisé (sous une forme à feuilles longues) dans les deux régions ; cette bimodalité de répartition a été signalée par HASLAM (1987).

\subsubsection{Le déterminisme des successions des phytocénoses}

Si, en milieu non pertubé, les successions des phytocénoses traduisent surtout la zonation longitudinale et les changements progressifs amont-aval des conditions morphodynamiques et trophiques des cours d'eau, ce n'est pas le cas lorsque des altérations importantes de la qualité de l'eau surviennent.

Ainsi, dans les Vosges du Nord, on observe une augmentation régulière, de $A$ à $D$, des teneurs en éléments nutritifs (phosphates et azote ammoniacal). Nous sommes donc en présence d'une séquence d'eutrophisation en eau faiblement minéralisée, vicariante écologique et analogue à celle mise au point pour les eaux carbonatées des rivières phréatiques du Ried alsacien (CARBIENER ot al., 1990). En cas d'eutrophisation anthropique importante dans les secteurs d'amont se traduisant par une apparition précoce des stades $C$ ou D (par exemple sur le Rothbach, fig. 2b), les caractères morphométriques (largeur, ordre de drainage, profondeur et vitesse de courant), et de minéralisation des eaux sont ceux des types qui existeraient s'il n'y avait pas d'apports anormaux. Ceci prouve bien que le déterminisme de ces groupements est avant tout d'ordre trophique, ce qui est particulièrement bien traduit par les phosphates.

En Bretagne, les variations de la végétation en fonction du niveau trophique apparaissent moins tranchées que dans les Vosges du Nord. En effet, dans les Vosges du Nord, la référence oligotrophe est forestière sans aucune influence des pratiques agricoles, ce qui n'est réalisé qu'en de très rares endroits dans le Massif armoricain. De ce fait, les communautés de type $A$ et $B$ y sont assez rares ; elles sont le plus souvent remplacées par des 
stades $A^{\prime}$ (groupements fontinaux à petites renoncules ou $M$. fontana) et $B^{\prime}$ (groupements à $A$. nodiflorum et/ou $G$. fluitans avec parfois $N$. officinale) traduisant une eutrophisation; on se situe fréquemment dans un stade $C$ plus à l'aval. Dans ces conditions de relative homogénéité trophique, les paramètres morphométriques apparaissent prédominants dans le déterminisme écologique des groupements, ainsi que cela a pu être établi par des analyses en composantes principales et des analyses factorielles des correspondances (HAURY, 1985 ; HAURY, 1990).

\subsection{Eléments pour l'élaboration d'un système d'indication biologique fondé sur la macrophyte}

La flore des eaux courantes est aisément repérable, sinon toujours facilement identifiable. Elle comprend un fond commun acidophile et des espèces plus ubiquistes quant au pH. Elle marque a la fois les conditions physiques du milieu (profondeur, éclairement, vitesse du courant, ...) et la qualité physico-chimique de l'eau. Par ailleurs, elle est diversifiée selon les régions, ce qui permet d'intégrer les variations biogéographiques de répartition des espèces et des communautés. De l'ensemble de ces caractères, il ressort que les macrophytes peuvent être utilisés comme des intégrateurs des conditions écologiques des cours d'eau à la fois pour la qualité de l'eau et les conditions hydrodynamiques locales (WHITTON, 1979 ; EMPAIN et al., 1980 ; MERIAUX et WATTEZ, 1980, ...), en complément des systèmes biotypologiques élaborés à l'aide des diatomées el des invertébrés benthiques. Dans ces systèmes biologiques, les macrophytes présentent l'avantage d'être assez aisément cartographiables et, intégrant les variations interannuelles de la qualité de l'eau, de permettre ensuite un suivi rapide et fiable de la qualité de l'eau à partir des espèces ou des groupements bioindicateurs (ici, principalement $\boldsymbol{P}$. polygonifolius, les différentes espèces de Callitriches et de Renoncules, $A$. riparium et $S$. undulata).

\section{RÉFÉRENCES BIBLIOGRAPHIQUES}

ABBAYES H. (Des), ClAUSTRES G., CORILLION R., DUPONT P., 1971. Flore et végétation du Massif amoricain. I - Flore vasculaire. Pres. univ. Bretagne, SaintBrieuc, $1226 \mathrm{p}$.

AUGIER J., 1966. Flore des bryophytes. Lechevalier, Paris, $702 \mathrm{p}$.

BUTCHER R.W., 1933. Studies on the ecology of rivers - 1: On the distribution of macrophytic vegetation in the rivers of Britain. J. Ecol. 21 : 58-91.
CARBIENER R. TREMOLIERES $M$. MERCIER J.L., ORSTCHEIT A., 1990. Aquatic macrophyte communities as bioindicators of eutrophication in calcareous oligosaprobe stream waters (Upper Rhine plain, Alsace). Vegetatio, 86 : 71-88.

CORLEY M.F.V., CRUNDWELL A.C., DULLL R., HILL M.O., SMITH A.J.E., 1982. Mosses of Europe and the Azores; an annotated list of species, with synonyms from the recent literature. J. Bryol., 11 (4) : 609-689. 
DETHIOUX M., NOIRFALISE A., 1985. Les groupements méophiles à renoncules aquatiques en moyenne et haute Belgique. Tuexenia, 5 : 31-39.

EMPAIN A., LAMBINON J., MOUVET C., KIRCHMANN R., 1980. Utilisation des bryophytes aquatiques et subaquatiques comme indicateurs biologiques de la qualité des eaux courantes. In P. Pesson (ed.) La pollution des eaux continentales incidences sur les biocénoses aquatiques (20 éd.) : 195-223, Gauthier Villars, Paris.

FABRI R., LECLERCQ L., 1977. Végétation et caractéristiques physico-chimiques des eaux de trois rivières de haute Ardenne (Belgique). Bull. Soc. roy. Bot. Belg., 110 : 202-216.

GEHU J.M., MERIAUX J.L., 1983. Distribution et caractères phytosociologiques des Renoncules du sous-genre Batrachium dans le Nord de la France. Bull. Soc. bot. Fr., Lettres bot., 130 (1) : 57-67.

GROLLE R., 1983. Hepatics of Europe including the Azores : an annotated list of species, with synonyms from the recent literature. J. Bryol., 12 (3) : 403.459.

HARTOG C. (Den), SEGAL S., 1964. A new classification of the water plant communities. Acta Bot. Neer. 13 : 367-393.

HASLAM S.M., 1978. River plants. Cambridge Univ. Press, Cambridge, 396 p.

HASLAM S.M., 1987. River plants of Western Europe. Cambridge Univ. Press, Cambridge, $512 \mathrm{p}$.

HASLAM S.M., MOLITOR A.M.M., 1988. The macrophytic vegetation of the major rivers of Luxembourg. Bull. Soc. Nat. luxemb., 88 : 3-54.

HASLAM S.M., SINKER C.A., WOLSELEY P.A., 1975. British water plants. Field Stud. $4:$ 243-351.

HAURY J., 1982. Quelques méthodes d'étude de la végétation macrophytique en écosystème dukçaquicole courant. Applica. tion au réseau hydrographique du Scorff, Bretagne. Sci. agron. Rennes 1982-2 : 17-33.

HAURY J., 1985. Etude écologique des macrophytes du Sconff (Bretagne-Sud). Thès. Doct.-ing. Ecol. Univ. Rennes I, $243 \mathrm{p}$.

HAURY J., 1988a. Macrophytes du Scorff : distribution des espèces et bio-typologie. Bull. Soc. Sci. Bretagne, 59 (1-4) : 53-66.
HAURY J., 1988b. Macrophytes du Trieux (Bretagne-Nord) : les ensembles floristiques. Bull. Soc. Sc. Nat. Ouest de la France, now. sér., 10 (3) : 135-150.

HAURY J., 1989. Macrophytes du Trieux (Bretagne-Nord) : II - Analyse des relations espèces-milieu physique par la méthode des profils écologiques. Bull. Soc. Sc. Nat. Ouest de la France, nouv. sér., 11 (4) : 193-207.

HAURY J., 1990. Macrophytes du Trieux (Bretagne-Nord) : III - Relations macrophytes-qualités des eaux. Bull. Soc. Sc. Nat. Ouest de la France, nouv. sér., 12 (4) : 141-154.

HOLMES N.T.H., 1983. Focus on Nature Conservancy. 4 - Typing British rivers according to their Flora. Nature Conservancy Council, Shrewbury. 194 p.

HOLMES N.T.H., WHITTON B.A., 1977. The macrophytic vegetation of the river Tees : observed and predicted changes. Freshwat. Biol. 7 : 43-60.

HUBSCHMANN A.V. (Von), 1957. Zur Systematik der Wassermoos-gesellschaten. Mitt. Florsit. Soziol. Arbeitsgem 6/7: 147-151.

ILLIES J., BOTOSANEANU L., 1963. Problème et méthode de la classification et de la zonation écologique des eaux courantes considérées surtout d'un point de vue faunistique. Mitt. internat. Verein Theor. Angew. Limnol., 12 : 1-57.

KOHLER A., 1975. Submerse Makrophyten und ihre gesellschaften als Indikatoren der gewasserbelastung. Beitr. Naturk. Forsch. Sudwestdeutschl., 34 : 149-159.

KOHLER A., VOLLRATH H., BEISL E., 1971. Zur Verbreitung, Vergesellschatung und Okologie des Gefass-Makrophyten im Fliesswassersystem Moosach (Münchener Ebene). Arch. Hydrobiol., 69 : 333-365.

LEGLIZE L., PELTRE M.C., DECLOUX J.P., DUVAL T., PARIS P., ZUMSTEIN J.F., 1990. Caractérisation des milieux aquatiques d'eaux courantes et végétation fixée. 14ème Conf. Int. COLUMA, Versailles 23-24 janvier 1990 : 237-245.

MARSTALLER R., 1987. Die Moosgesellschaften der Klasse PlatyhypnidioFontinaletea antipyreticae Philippi 1956 30 Beitrag zur Moosvegetation Thüringens. Phytocoenologia, 15 (1) : 85-138. 
MERIAUX J.L., 1982. L'utilisation des macrophytes des phytocénoses aquatiques comme indicateurs de la qualité des eaux. Naturalistes Belges, 63 : 18-24.

MERIAUX J.L., WATTEZ J.R., 1980. Les végétations aquatiques et subaquatiques : relations avec la qualité des eaux. In P. Pesson (ed.). La pollution des eaux continentales - Incidences sur les biocénoses aquatiques ( $2^{\theta}$ éd.) : 225-242, Gauthier Villars, Paris.

MULLER S., 1990. Une séquence de groupements végétaux bio-indicateurs d'eutrophisation croissante des cours d'eau faiblement minéralisés des Basses Vosges gréseuses du Nord. C. R. Acad. Sci. Paris, 310, Sér. III : 509-514.

OBERDORFER E., 1977. Süddeutsche Pflanzengesellschaten - I ( $2^{\circ}$ ed.). Fischer, Stuttgart, $311 \mathrm{p}$.

PHILIPPI G., 1956. Einige Moosgesellschaften des Sudschwarzwaldes und der angrenzenden Rheinebene. Beitr. Naturkd. Forsch. SudwestDeutschl., 15 : 91-124.

SMITH A.J.E., 1980. The moss flora of Britain and Ireland. ( $2^{\circ}$ éd.), Cambridge Univ. Press, Cambridge, $706 \mathrm{p}$.

TUTIN T.G., HEYWOOD V.H., BURGES N.A., MOORE D.M., VALENTINE D.H., WALTERS S.M., WEBB D.A. (Eds) 1964 à
1980 - Flora europaea. 5 vol., 1 : 1964, 464 p.; 2 : 1968, 455 p. : 3 : 1972, 385 p. ; $4: 1976,405$ p. ; 5 : 1980, 452 p., Cambridge University Press, Cambridge.

WHITTON B.A., 1979. Plants as indicators of river water quality. In James A., Evison L. (Eds.) Biological indicators of water quality: $5,1-5,34$. Wiley \& Sons Ed., Chichester.

WIEGLEB G., 1983a. Recherches méthodologiques sur les groupements végétaux des eaux courantes. In Gehu J.M. (ed.) Les végétations aquatiques et amphibies : 69-83. Cramer, Vaduz.

WIEGLEB G., 1983b. A phytosociological study of the macrophytic vegetation of running waters in Western Lower Saxony (Fed. Repub. Ger.). Aquat. Bot., $17: 251$ 274.

WIEGLEB G., 1988. Analysis of flora and vegetation in rivers : concepts and application. In Symoens J.J. (ed.) Vegetation of inland waters: 311-378, Kluwer Academic Publishers, Dordrecht (Netherlands).

WOLFF C., 1987. Analyse de la végétation aquatique et de la végétation riveraine de la Haute-Süre en fonction des perturbations du milieu. Bull. Soc. Nat. luxemb., 87 : 1-52. 